\title{
MAD families with strong combinatorial properties
}

\author{
by \\ Jörg Brendle and Greg Piper (Kobe)
}

\begin{abstract}
In his paper in Fund. Math. 178 (2003), Miller presented two conjectures regarding MAD families. The first is that $\mathrm{CH}$ implies the existence of a MAD family that is also a $\sigma$-set. The second is that under $\mathrm{CH}$, there is a MAD family concentrated on a countable subset. These are proved in the present paper.
\end{abstract}

1. Introduction. Let $[\omega]^{\omega}$ denote the infinite subsets of the natural numbers $\omega$. Two sets $a, b \in[\omega]^{\omega}$ are almost disjoint if $a \cap b$ is finite. A family $\mathcal{A} \subseteq[\omega]^{\omega}$ is almost disjoint if all its members are pairwise almost disjoint, and maximal almost disjoint (a MAD family, for short) if for all $x \in[\omega]^{\omega}$, $a \cap x$ is infinite for some $a \in \mathcal{A}$. In our work we construct MAD families with additional strong topological properties.

We think of MAD families as sets of reals and, accordingly, we identify elements of $[\omega]^{\omega}$ with their characteristic functions, i.e., with elements of $2^{\omega}$ which are not eventually 0 . Conversely, we usually equate $a \in 2^{\omega}$ with the corresponding set $\{i \in \omega: a(i)=1\}$.

An uncountable set of reals $X \subseteq 2^{\omega}$ is a $Q$-set if every subset of $X$ is a relative $G_{\delta}$ set, and a $\sigma$-set if every relative Borel subset is a relative $G_{\delta}$ set, i.e., for all Borel $B \subseteq 2^{\omega}$ there is a $G_{\delta}$ set $G \subseteq 2^{\omega}$ such that $B \cap X=G \cap X$. Every $Q$-set is a $\sigma$-set. Miller [8, Theorem 1] proved it is consistent with ZFC that there is a MAD $Q$-set. Such a set necessarily has size less than $\mathfrak{c}=\left|2^{\omega}\right|$. A modification of his argument showed it is consistent

2000 Mathematics Subject Classification: Primary 03E50; Secondary 03E05, 03E15, $03 \mathrm{E} 40$.

Key words and phrases: MAD family, $\sigma$-set.

Research of J. Brendle was partially supported by Grant-in-Aid for Scientific Research (C) 17540116, Japan Society for the Promotion of Science. The author wishes to thank Sun Yat-Sen University in Guangzhou, China, for its support, and Yi Zhang for his hospitality during September 2005 when part of this research was carried out.

Research of G. Piper was supported by JSPS Postdoctoral Fellowship for Foreign Researchers ID P 04048 and by Grant-in-Aid for Scientific Research No. 16.04048, Japan Society for the Promotion of Science. 
that there is a MAD $\sigma$-set of size $\mathfrak{c}$ where $\mathfrak{c}$ can be arbitrary [8, Theorem 3]. The existence of a MAD $\sigma$-set is a $\Sigma_{1}^{2}$ sentence. Thus, by Woodin's $\Sigma_{1}^{2}$ absoluteness (see [3, Theorem 3.2.1]) which asserts that if $\kappa$ is a measurable Woodin cardinal, $\mathrm{CH}$ holds and $\mathbb{P}$ is a forcing notion of size less than $\kappa$ then any $\Sigma_{1}^{2}$ sentence true in $V^{\mathbb{P}}$ is also true in $V$, Miller deduced that there is a MAD $\sigma$-set under $\mathrm{CH}+$ there is a measurable Woodin cardinal $[8$, Remark on p. 279]. Accordingly he conjectured such a set could be constructed under $\mathrm{CH}$ alone [8, Conjecture 4]. We prove this is indeed the case.

TheOREM 1. CH implies there is a MAD $\sigma$-set.

Note that some assumption is necessary because there may be no $\sigma$-set [5, Theorem 22].

A set of reals $X \subseteq 2^{\omega}$ is concentrated on $Y \subseteq 2^{\omega}$ if for any open $U \supseteq Y$, $X \backslash U$ is at most countable. Miller [8, Theorem 5] also proved the generic MAD family adjoined by Hechler's standard forcing notion [1] is concentrated on a countable subset of itself. Using the same large cardinal considerations, he conjectured such a MAD family existed under $\mathrm{CH}[8$, Conjecture 7]. We confirm this.

THEOREM 2. CH implies that there exists an infinite MAD family which is concentrated on a countable subset of itself.

Again, this is not true in ZFC alone because all concentrated sets may be countable. (The latter holds, e.g., in Laver's model for the Borel conjecture [4] because every set concentrated on a countable set has strong measure zero [6, Theorem 3.1].)

We prove Theorem 2 in Section 2 by singling out one property of the generic MAD family (of [1]) used to prove Theorem 5 of [8] and then setting up a recursive construction which preserves this property along with creating a MAD family and turning it into a concentrated set. The proof of Theorem 1 is much harder. We use a topological argument, successively creating the members of the MAD family $\mathcal{A}$ as Cohen reals in an appropriate Polish space, roughly, the space of reals almost disjoint from previous members of $\mathcal{A}$, equipped with a natural topology finer than the standard topology. This approach has two advantages. First, we get maximality for free because for each real $x$, the set $G_{x}$ of reals which have infinite intersection with $x$ is a dense $G_{\delta}$ set, even in the finer topology. Second, we guarantee that the set of reals $V_{\alpha}$ on which a given Borel set $B_{\alpha}$ and a generically adjoined $G_{\delta}$ set $U_{\alpha}$ agree is also a dense $G_{\delta}$ set. Thus any later member of $\mathcal{A}$ will belong either to both $B_{\alpha}$ and $U_{\alpha}$ or to neither, and $B_{\alpha} \cap \mathcal{A}=U_{\alpha} \cap \mathcal{A}$ will follow. See Section 3 for details. In Section 4 , we briefly discuss generalizations of our results under Martin's Axiom MA. 
2. A MAD family concentrated on a countable subset. In this section, we prove Theorem 2. Assuming $\mathrm{CH}$, we define a suitable MAD family inductively.

First choose an almost disjoint family $\left\langle a_{n}: n<\omega\right\rangle$ with the following property:

$$
\begin{aligned}
\forall F \subseteq \omega \text { finite } \forall s \in 2^{<\omega} \exists n & <\omega \\
& \left(s \subseteq a_{n} \text { and } \bigcup\left\{a_{m}: m \in F\right\} \cap a_{n} \subseteq|s|\right) .
\end{aligned}
$$

This technical property is a strengthening of denseness and is needed in the inductive definition of the MAD family. It is easy to construct such a family. In fact, the standard forcing for adding a countable almost disjoint family [1] generically adds $a_{n}$ satisfying $(\star)$.

We now proceed by induction to produce $a_{\alpha}$ for $\omega \leq \alpha<\omega_{1}$. Let $\left\langle U_{\alpha}\right.$ : $\left.\alpha<\omega_{1}\right\rangle$ list all the open subsets of $2^{\omega}$ that contain all of the $a_{n}$. That is, $\left\{a_{n}: n<\omega\right\} \subseteq U_{\alpha}$ for all $\alpha<\omega_{1}$. Let $\left\langle r_{\alpha}: \omega \leq \alpha<\omega_{1}\right\rangle$ list all the infinite elements of $2^{\omega}$.

We construct $a_{\alpha}, \alpha \geq \omega$, satisfying the following conditions.

(1) $\forall \beta<\alpha\left(\left|a_{\beta} \cap a_{\alpha}\right|<\aleph_{0}\right)$.

(2) $\forall \beta<\alpha\left(a_{\alpha} \in U_{\beta}\right)$.

(3) $\exists \beta \leq \alpha\left(\left|r_{\alpha} \cap a_{\beta}\right|=\aleph_{0}\right)$.

(4) $\forall F \subseteq \alpha+1$ finite $\forall s \in 2^{<\omega} \exists n<\omega\left(s \subseteq a_{n}\right.$ and $\bigcup\left\{a_{\beta}: \beta \in F\right\} \cap a_{n}$ $\subseteq|s|)$.

The last condition is the analogue of $(\star)$ above. Notice that (1) and (4) hold for $\alpha<\omega$ by construction. (Properties (2) and (3) are irrelevant for $\alpha<\omega$.)

We construct $a_{\alpha}$ by recursively producing countably many of its initial segments $s_{j}, j<\omega$, with $\left|s_{j}\right| \geq j$ as well as finite sets $X_{j}, j<\omega$, which will identify the $a_{\beta}$ that must be avoided when we extend $s_{j}$. Let $\left\langle\beta_{j}: j<\omega\right\rangle$ enumerate $\alpha$, and let $\left\langle\left(t_{j}, F_{j}\right): j<\omega\right\rangle$ list all the pairs in $2^{<\omega} \times[\alpha]<\omega$ in such a way that $\left|t_{j}\right| \leq j$. As usual, $[s]=\left\{y \in 2^{\omega}: s \subseteq y\right\}$ denotes the clopen set defined by $s \in 2^{<\omega}$.

Stage 0. Let $s_{0} \in 2^{<\omega}$ be such that $\left[s_{0}\right] \subseteq U_{\beta_{0}}$. Let $X_{0}=\left\{a_{\beta_{0}}\right\}$.

STAGE $k+1$. Assume that we have already defined $s_{j}$ and $X_{j}$ for all $j \leq k$ such that $\left[s_{j}\right] \subseteq U_{\beta_{j}}$ and $a_{\beta_{j}} \in X_{j}$. We define $s_{k+1} \supseteq s_{k+1}^{\prime} \supseteq s_{k}$ and $X_{k+1} \supseteq X_{k}$.

First we take care of property (2). Since $X_{k}$ is finite, by the inductive hypothesis (using (4)) we can find $n_{k}<\omega$ such that $s_{k} \subseteq a_{n_{k}}$ and $\bigcup X_{k} \cap a_{n_{k}} \subseteq\left|s_{k}\right|$. Now let $j_{k} \geq \max \left\{k+1,\left|s_{k}\right|\right\}$ be such that $\left[a_{n_{k}}\left\lceil j_{k}\right] \subseteq\right.$ $U_{\beta_{k+1}}$. There must be such an $j_{k}$ since $a_{n_{k}} \in U_{\beta_{k+1}}$. Let $s_{k+1}^{\prime}=a_{n_{k}}\left\lceil j_{k}\right.$. 
Note that this will imply that once we have defined $s_{j}$ for all $j<\omega$, $a_{\alpha}=\bigcup\left\{s_{j}: j<\omega\right\} \in U_{\beta_{k+1}}$.

Next we ensure that (4) holds for finite sets containing $\alpha$. By the inductive hypothesis, we know that (4) holds for finite $F \subseteq \alpha$. Let $F_{k}^{\prime}=F_{k} \cup\left\{n_{k}\right\}$. Given $t_{k}$ and $F_{k}^{\prime}$, there is $i_{k}$ such that $t_{k} \subseteq a_{i_{k}}$ and $\bigcup\left\{a_{\beta}: \beta \in F_{k}^{\prime}\right\} \cap a_{i_{k}} \subseteq$ $\left|t_{k}\right|$. To obtain $\bigcup\left\{a_{\beta}: \beta \in F_{k} \cup\{\alpha\}\right\} \cap a_{i_{k}} \subseteq\left|t_{k}\right|$ it suffices to ensure that $a_{\alpha} \cap a_{i_{k}}=a_{n_{k}} \cap a_{i_{k}}$. We achieve this simply by adding $a_{i_{k}}$ to $X_{k}$. Thus, let $X_{k+1}=X_{k} \cup\left\{a_{\beta_{k+1}}\right\} \cup\left\{a_{i_{k}}\right\}$.

If $r_{\alpha} \cap a_{\beta}$ is infinite for some $\beta<\alpha$, let $s_{k+1}=s_{k+1}^{\prime}$. If not, we need to guarantee condition (3) as well: let $i \geq\left|s_{k+1}^{\prime}\right|$ be minimal such that $r_{\alpha}(i)=1$ but $a(i)=0$ for all $a \in X_{k+1}$. There must be such an $i$ since $r_{\alpha}$ is infinite and almost disjoint from all of the elements of $X_{k+1}$. Let $s_{k+1}$ be a sequence of length $i+1$ extending $s_{k+1}^{\prime}$ and with $s_{k+1}(i)=1$ and $s_{k+1}(j)=0$ for $\left|s_{k+1}^{\prime}\right| \leq j<i$. Note that this will imply that $a_{\alpha}=\bigcup\left\{s_{j}: j<\omega\right\}$ and $r_{\alpha}$ will have infinite intersection.

Once we have defined $s_{j}$ for all $j<\omega$, we let $a_{\alpha}=\bigcup\left\{s_{j}: j<\omega\right\}$. We must now check that each of the conditions (1)-(4) hold.

(1) By construction, for all $k<\omega, a_{\beta_{k}} \cap a_{\alpha} \subseteq\left|s_{k}\right|$ because $a_{\beta_{k}} \in X_{k}$ and $X_{k}$ is the set of reals which are avoided when extending $s_{k}$.

(2) and (3) are immediate as we observed during the induction.

(4) This follows from the fact that we put $a_{i_{k}}$ into $X_{k+1}$. Indeed, the latter implies $a_{\alpha} \cap a_{i_{k}} \subseteq\left|s_{k+1}^{\prime}\right|$. Also $a_{\alpha}\left\lceil j_{k}=s_{k+1}^{\prime}=a_{n_{k}}\left\lceil j_{k}\right.\right.$ where $j_{k} \geq k+1$, and $a_{n_{k}} \cap a_{i_{k}} \subseteq\left|t_{k}\right| \leq k$. Thus $a_{\alpha} \cap a_{i_{k}}=a_{n_{k}} \cap a_{i_{k}}$ and we remarked earlier this was exactly what was needed to ensure (4).

This completes the recursive construction of the family $\left\langle a_{\alpha}: \alpha<\omega_{1}\right\rangle$. By (1), it is an almost disjoint family, by (3) it is maximal, and by (2) it is concentrated on $\left\{a_{n}: n<\omega\right\}$ because for any $\alpha<\omega_{1},\left\{\beta<\omega_{1}\right.$ : $\left.a_{\beta} \notin U_{\alpha}\right\} \subseteq \alpha+1 \backslash \omega$, which is countable. This completes the proof of Theorem 2 .

\section{CH implies there exists a MAD $\sigma$-set}

3.1. The framework of the proof. Before going into the actual combinatorial details, we describe the framework of the proof of Theorem 1 .

Assume we have models $\left\langle M_{\alpha}: \alpha<\omega_{1}\right\rangle$ and $\left\langle N_{\alpha}: \alpha<\omega_{1}\right\rangle$ of ZFC such that:

- $M_{\alpha} \subseteq N_{\alpha} \subseteq M_{\alpha+1}$,

- $M_{\alpha}$ is countable in $N_{\alpha}$,

- $\alpha$ is countable in $M_{\alpha}$ and all $N_{\beta}, \beta<\alpha$, are countable in $M_{\alpha}$ (so $\left\langle N_{\beta}: \beta<\alpha\right\rangle \in M_{\alpha}$ is countable in $M_{\alpha}$ ),

- $2^{\omega} \subseteq \bigcup_{\alpha<\omega_{1}} M_{\alpha}=\bigcup_{\alpha<\omega_{1}} N_{\alpha}$. 
We shall build perfect Polish spaces $\left(X_{\alpha}, \mathcal{S}_{\alpha}\right),\left(Y_{\alpha}, \mathcal{T}_{\alpha}\right)$ (where $\mathcal{S}_{\alpha}, \mathcal{T}_{\alpha}$ denote the respective topologies) such that:

- $X_{\alpha+1} \subseteq Y_{\alpha} \subseteq X_{\alpha}$

- $X_{\alpha}=\bigcap_{\beta<\alpha} X_{\beta}=\bigcap_{\beta<\alpha} Y_{\beta}=Y_{\alpha}$ for limit $\alpha$,

- the topology $\mathcal{T}_{\alpha}$ refines the topology $\mathcal{S}_{\alpha}$ (restricted to $Y_{\alpha}$ ),

- the topology $\mathcal{S}_{\alpha+1}$ refines the topology $\mathcal{T}_{\alpha}$ (restricted to $X_{\alpha}$ ),

- $\mathcal{T}_{\alpha}=\bigcup_{\beta<\alpha} \mathcal{T}_{\beta}=\bigcup_{\beta<\alpha} \mathcal{S}_{\beta}=\mathcal{S}_{\alpha}$ for limit $\alpha$,

- $Y_{\alpha} \in M_{\alpha}, X_{\alpha+1} \in N_{\alpha}$,

- $X_{0}=Y_{0}=2^{\omega}$ and $\mathcal{S}_{0}=\mathcal{T}_{0}=$ the standard topology.

Notice that for limit $\alpha$, we indeed have $Y_{\alpha}=\bigcap_{\beta<\alpha} X_{\beta} \in M_{\alpha}$ because $\left\langle N_{\beta}: \beta<\alpha\right\rangle \in M_{\alpha}$. While we are mainly interested in $\left(X_{\alpha}, \mathcal{S}_{\alpha}\right)$ and $\left(Y_{\alpha}, \mathcal{T}_{\alpha}\right)$, we shall often think of $\mathcal{S}_{\alpha}$ and $\mathcal{T}_{\alpha}$ as refining the standard topology on $2^{\omega}$.

More explicitly, there will be sets $F_{\alpha}^{s}, F_{\alpha}, H_{\alpha}^{n}, H_{\alpha} \subseteq 2^{\omega}\left(s \in 2^{<\omega}, n \in \omega\right)$ such that:

- all $F_{\alpha}^{s}, H_{\alpha}^{n}$ are closed in the standard topology,

- $F_{\alpha}=\bigcup_{s \in 2<\omega} F_{\alpha}^{s}$ and $H_{\alpha}=\bigcup_{n \in \omega} H_{\alpha}^{n}$ are $F_{\sigma}$ sets,

- $X_{\alpha+1}=Y_{\alpha} \cap F_{\alpha}$,

- the topology $\mathcal{S}_{\alpha+1}\left(\right.$ on $X_{\alpha+1}$ ) is generated by (the restriction of) $\mathcal{T}_{\alpha} \cup$ $\left\{F_{\alpha}^{s}: s \in 2^{<\omega}\right\}$ (so all the sets $F_{\alpha}^{s}$ are made clopen).

The description of the space $Y_{\alpha+1}$ and its topology $\mathcal{T}_{\alpha+1}$ is somewhat more difficult. There are closed $P_{\alpha} \subseteq X_{\alpha+1}$ and open $O_{\alpha} \subseteq X_{\alpha+1}$ (in the topology $\left.\mathcal{S}_{\alpha+1}\right)$ such that:

- $P_{\alpha} \cup O_{\alpha}=X_{\alpha+1}$,

- $P_{\alpha} \cap O_{\alpha}=\emptyset$,

- $Y_{\alpha+1}=P_{\alpha} \cup\left(X_{\alpha+1} \cap H_{\alpha}\right)=P_{\alpha} \cup\left(O_{\alpha} \cap H_{\alpha}\right)$,

- the topology $\mathcal{T}_{\alpha+1}\left(\right.$ on $\left.Y_{\alpha+1}\right)$ is generated by (the restriction of) $\mathcal{S}_{\alpha+1}$ as well as sets of the form $F \cap H_{\alpha}^{n}$ where $F \cap X_{\alpha+1} \subseteq O_{\alpha}$ and $F$ is open in $\mathcal{S}_{\alpha+1}$ and $n \in \omega$.

The latter stipulation means that the family $\mathcal{F}_{\alpha+1}$ of sets $F \in \mathcal{S}_{\alpha+1}$ with $F \cap X_{\alpha+1} \subseteq P_{\alpha}$ and of sets $F \cap \bigcap_{j<m} H_{\alpha}^{n_{j}}$ with $F \cap X_{\alpha+1} \subseteq O_{\alpha}$ and $F \in \mathcal{S}_{\alpha+1}$ is dense in the topology $\mathcal{T}_{\alpha+1}$. That is, $\mathcal{F}_{\alpha+1} \subseteq \mathcal{T}_{\alpha+1}$ and every $F \in \mathcal{T}_{\alpha+1}$ contains a member of $\mathcal{F}_{\alpha+1}$.

Also notice that, more generally, the topology $\mathcal{S}_{\alpha}$ is generated by the standard clopen sets together with $F_{\beta}^{s}\left(s \in 2^{<\omega}, \beta<\alpha\right)$ and certain (not all!) intersections of the latter sets with sets of the form $H_{\beta}^{n}(n \in \omega, \beta<$ $\alpha-1$ ), where for limit $\alpha$ we set $\alpha-1=\alpha$. Similarly $\mathcal{T}_{\alpha}$ is generated by the standard clopen sets together with $F_{\beta}^{s}\left(s \in 2^{<\omega}, \beta<\alpha\right)$ and some (not all!) intersections of the latter with $H_{\beta}^{n}(n \in \omega, \beta<\alpha)$. 
Observation 3.1. The spaces $\left(X_{\alpha}, \mathcal{S}_{\alpha}\right)$ and $\left(Y_{\alpha}, \mathcal{T}_{\alpha}\right)$ are indeed Polish.

Proof. By the characterization of $\mathcal{S}_{\alpha}$ directly preceding 3.1, all basic open sets of $\mathcal{S}_{\alpha}$ are closed in the standard topology $\mathcal{S}_{0}$. This means that by [2, Lemmata 13.2 and 13.3], $\left(X_{0}, \mathcal{S}_{\alpha}\right)$ is Polish. Similarly for the $\mathcal{T}_{\alpha}$. We prove by induction on $\beta \leq \alpha$ that all $\left(Z_{\beta}, \mathcal{U}_{\alpha}\right)$ are Polish as well where $Z \in\{X, Y\}$ and $\mathcal{U} \in\{\mathcal{S}, \mathcal{T}\}$ (and $\left(Y_{\beta}, \mathcal{S}_{\alpha}\right)$ is only considered for $\beta \leq \alpha-1$ ).

If $\beta=\gamma+1$ is successor, $\left(X_{\beta}, \mathcal{U}_{\alpha}\right)$ is Polish because $X_{\beta}=Y_{\gamma} \cap F_{\gamma}$ is open in $\left(Y_{\gamma}, \mathcal{U}_{\alpha}\right)$. Similarly, $P_{\gamma}$ is closed and $O_{\gamma} \cap H_{\gamma}$ is open in $\left(X_{\beta}, \mathcal{U}_{\alpha}\right)$. So $Y_{\beta}$ is $G_{\delta}$ in $\left(X_{\beta}, \mathcal{U}_{\alpha}\right)$ and thus Polish [2, Theorem 3.11] (here $\mathcal{U}=\mathcal{T}$ in case $\beta=\alpha)$.

Let $\beta$ be a limit ordinal. Since all $\left(X_{\gamma}, \mathcal{U}_{\alpha}\right), \gamma<\beta$, are Polish, the $X_{\gamma}$ form a decreasing sequence of $G_{\delta}$ subsets of $\left(X_{0}, \mathcal{U}_{\alpha}\right)$, their intersection $X_{\beta}=$ $\bigcap_{\gamma<\beta} X_{\gamma}$ is still such a $G_{\delta}$, and thus $\left(X_{\beta}, \mathcal{U}_{\alpha}\right)$ is Polish [2, Theorem 3.11].

We shall see below (Lemma 3.5) that all $\left(X_{\alpha}, \mathcal{S}_{\alpha}\right)$ and $\left(Y_{\alpha}, \mathcal{T}_{\alpha}\right)$ are also perfect.

Let $i_{n}=2^{n}$ and put $I=\left\{i_{n}: n \in \omega\right\}$. (In fact, the exact nature of the $i_{n}$ is irrelevant; what we need is that the sequence of $i_{n}$ is increasing very fast.) Clearly $I \in M_{0}$. We will have sets $U_{\alpha}^{n} \subseteq 2^{\omega}(n \in \omega)$ and $U_{\alpha}$ such that:

- $U_{\alpha}^{n}=2^{\omega} \backslash H_{\alpha}^{n}$ is open,

- $U_{\alpha}=2^{\omega} \backslash H_{\alpha}$, i.e. $U_{\alpha}=\bigcap_{n \in \omega} U_{\alpha}^{n}$ is $G_{\delta}$,

- each $U_{\alpha}^{n}$ is a union of basic clopen sets $\left[s_{\alpha}^{n, j}\right], j \in \omega$, such that:

$-\left|s_{\alpha}^{n, j}\right| \in I,\left|s_{\alpha}^{n, j}\right| \geq i_{n+j}$,

- for each $k \in \omega$, there is at most one $s_{\alpha}^{n, j}$ such that $\left|s_{\alpha}^{n, j}\right|=i_{k}$ (so $k \geq n+j)$,

- if $\left|s_{\alpha}^{n, j}\right|=i_{k}$ then there is $l \in\left(i_{k-1}, i_{k}\right)$ such that $s_{\alpha}^{n, j}(l)=1$.

3.2. The MAD family $\mathcal{A}=\left\{a_{\alpha}: \alpha<\omega_{1}\right\}$ (construction of the space $\left.X_{\alpha+1}\right)$. We come now to the details of the construction. We begin with the construction of the space $X_{\alpha+1}$ and associated objects.

For each $\alpha$ let $a_{\alpha}$ be a Cohen-generic real belonging to the space $Y_{\alpha}$ over the model $M_{\alpha}$ in the model $N_{\alpha}$ (i.e. $M_{\alpha}\left[a_{\alpha}\right] \subseteq N_{\alpha}$ ). Such an $a_{\alpha}$ clearly exists because $M_{\alpha}$ is countable in $N_{\alpha}$.

We let $F_{\alpha}^{s}=\left\{y: s \subseteq y\right.$ and $\left.(\forall l \geq|s|)\left(a_{\alpha}(l)=1 \Rightarrow y(l)=0\right)\right\}$, the set of reals $y$ which contain $s$ as an initial segment and which are disjoint from $A_{\alpha}$ beyond $|s|$. This is clearly closed, as required. Note also that $F_{\alpha}^{s} \subseteq[s]$.

Let $F_{\alpha}=\bigcup_{s \in 2<\omega} F_{\alpha}^{s}$, the set of reals almost disjoint from $a_{\alpha}$. Define $X_{\alpha+1}$ and $\mathcal{S}_{\alpha+1}$ as stipulated earlier.

LEMma 3.2. $\left\{a_{\alpha}: \alpha<\omega_{1}\right\}$ is an almost disjoint family. 
Proof. For $\beta<\alpha, F_{\beta}$ is the set of reals almost disjoint from $a_{\beta}$. Since $Y_{\alpha} \subseteq X_{\alpha} \subseteq F_{\beta}$ by construction, $Y_{\alpha}$ only contains reals almost disjoint from $a_{\beta}$. Thus $a_{\alpha}$ is almost disjoint from $a_{\beta}$.

Observation 3.3. A typical basic open set of $\left(X_{\alpha}, \mathcal{S}_{\alpha}\right)$ is of the form $\bigcap_{j<m_{0}} F_{\beta_{j}}^{s} \cap \bigcap_{j<m_{1}} H_{\gamma_{j}}^{n_{j}} \neq \emptyset\left(s \in 2^{<\omega}, n_{j} \in \omega, \beta_{j}<\alpha, \gamma_{j}<\alpha-1\right)$. Similarly for $\left(Y_{\alpha}, \mathcal{T}_{\alpha}\right)$.

Proof. Since $F_{\beta}^{s} \subseteq[s]$, there is no need to consider basic clopen sets of the standard topology, and a typical basic clopen set is of the form $F=$ $\bigcap_{j<m_{0}} F_{\beta_{j}}^{s_{j}} \cap \bigcap_{j<m_{1}} H_{\gamma_{j}}^{n_{j}} \neq \emptyset$. For $j, j^{\prime}<m_{0}$ we must have $s_{j} \subseteq s_{j^{\prime}}$ or $s_{j^{\prime}} \subseteq s_{j}$. Put $s=\bigcup_{j<m_{0}} s_{j}$. Clearly $F \subseteq[s]$. Since $F_{\beta_{j}}^{s_{j}} \cap[s] \neq \emptyset$, we must have $F_{\beta_{j}}^{s} \subseteq F_{\beta_{j}}^{s_{j}}$. In fact, $F_{\beta_{j}}^{s} \cap F=F_{\beta_{j}}^{s_{j}} \cap F$. Thus $F=\bigcap_{j<m_{0}} F_{\beta_{j}}^{s} \cap \bigcap_{j<m_{1}} H_{\gamma_{j}}^{n_{j}}$.

The following is crucial for several subsequent results (see Lemmata 3.5 and 3.6).

Lemma 3.4. Given any $m, \beta_{j}<\omega_{1}(j<m), n$ and $k$, there is $l \geq k$ such that $\left|\left(i_{l}, i_{l+1}\right) \backslash \bigcup_{j<m} a_{\beta_{j}}\right| \geq n$.

Proof. This is a standard Cohen-genericity argument, using the fact that $I \in M_{0}$. Fix $n$. We proceed by induction on $m$. Let $\beta_{j}, j<m$, be given such that $\beta_{0}<\beta_{1}<\cdots<\beta_{m-1}$. Assume the statement is true for $m-1$ for all $k$. Put $\alpha=\beta_{m-1}$. Then $a_{\beta_{j}} \in M_{\alpha}$ for $j<m-1$ and $a_{\alpha}=a_{\beta_{m-1}}$ is Cohen-generic over $M_{\alpha}$ in $Y_{\alpha}$. By 3.3, a typical basic open set of the topology $\mathcal{T}_{\alpha}$ (equivalently, condition in the Cohen forcing) is of the form $p=\bigcap_{j<m_{0}} F_{\gamma_{j}}^{s} \cap \bigcap_{j<m_{1}} H_{\delta_{j}}^{n_{j}} \neq \emptyset$. Without loss of generality, we assume $|s| \in I$. Apply the induction hypothesis with $k$ replaced by $\max \{|s|, k\}$ and find $l \geq \max \{|s|, k\}$ with $\left|\left(i_{l}, i_{l+1}\right) \backslash \bigcup_{j<m-1} a_{\beta_{j}}\right| \geq n$. Notice that $|s| \leq l<i_{l}$. Thus, we may strengthen the condition, replacing $s$ by $t \supseteq s$ such that $|t|=i_{l+1}$ and $t(i)=0$ for $i \in\left[|s|, i_{l+1}\right)$, to get $q=\bigcap_{j<m_{0}} F_{\gamma_{j}}^{t} \cap \bigcap_{j<m_{1}} H_{\delta_{j}}^{n_{j}}$. To see that this works, notice that by the definition of the $F_{\gamma_{j}}^{s}$ and $H_{\delta_{j}}^{n_{j}}$, we must indeed have $[t] \cap \bigcap_{j<m_{0}} F_{\gamma_{j}}^{s} \cap \bigcap_{j<m_{1}} H_{\delta_{j}}^{n_{j}} \neq \emptyset$. The stronger condition $q$ clearly forces $\left|\left(i_{l}, i_{l+1}\right) \backslash\left(\bigcup_{j<m-1} a_{\beta_{j}} \cup \dot{a}_{\alpha}\right)\right| \geq n$ so we are done.

Lemma 3.5. All spaces $\left(X_{\alpha}, \mathcal{S}_{\alpha}\right)$ and $\left(Y_{\alpha}, \mathcal{T}_{\alpha}\right)$ are perfect Polish spaces.

Proof. We already observed that $\mathcal{S}_{\alpha}$ and $\mathcal{T}_{\alpha}$ were Polish. So it suffices to show $X_{\alpha}$ and $Y_{\alpha}$ are perfect. Consider $X_{\alpha}$, and let $\left\{\beta_{j}: j \in \omega\right\}=\alpha$, $\left\{\gamma_{j}: j \in \omega\right\}=\alpha-1$. Recursively construct $\ell_{j}, n_{j} \in \omega$ and $s_{j}(\tau) \in 2^{<\omega}$ $\left(\tau \in 2^{j}\right)$ such that

- $\ell_{j}<\ell_{j^{\prime}}$ for $j<j^{\prime}$

- $\left|s_{j}(\tau)\right|=i_{\ell_{j}}$, 
- if $j \leq j^{\prime}$ and $\tau \subseteq \tau^{\prime}, \tau \in 2^{j}, \tau^{\prime} \in 2^{j^{\prime}}$, then $s_{j}(\tau) \subseteq s_{j^{\prime}}\left(\tau^{\prime}\right), s_{j^{\prime}}\left(\tau^{\prime}\right) \in$ $F_{\beta_{j}}^{s_{j}(\tau)}$ and $s_{j^{\prime}}\left(\tau^{\prime}\right) \in H_{\gamma_{j}}^{n_{j}}$.

Here, $s \in F_{\beta_{j}}^{s_{j}(\tau)}\left(s \in H_{\gamma_{j}}^{n_{j}}\right.$, respectively) means that $s$ belongs to the tree defining the closed set $F_{\beta_{j}}^{s_{j}(\tau)}\left(H_{\gamma_{j}}^{n_{j}}\right.$, resp.).

For $j=0$, let $\ell_{0}=0$, choose $s_{0}(\langle\rangle)$ of length $i_{0}=2^{0}=1$ arbitrary and let $n_{0}$ be such that $s_{0}(\langle\rangle) \in H_{\gamma_{0}}^{n_{0}}$.

Suppose $\ell_{j}, n_{j}$, and $s_{j}(\tau)$ have been defined. By Lemma 3.4, we can choose $\ell_{j+1}>\ell_{j}$ such that $\left|\left(i_{\ell_{j+1}-1}, i_{\ell_{j+1}}\right) \backslash \bigcup_{j^{\prime} \leq j} a_{\beta_{j^{\prime}}}\right| \geq j+2$. Set $A=$ $\left(i_{\ell_{j+1}-1}, i_{\ell_{j+1}}\right) \backslash \bigcup_{j^{\prime} \leq j} a_{\beta_{j^{\prime}}}$. Fix $\tau \in 2^{j}$. Let $T_{\tau}=\left\{s: s_{j}(\tau) \subseteq s,|s|=i_{\ell_{j+1}}\right.$ and $\left.\forall i \in|s| \backslash\left(\left|s_{j}(\tau)\right| \cup A\right)(s(i)=0)\right\}$. Clearly $\left|T_{\tau}\right| \geq 2^{j+2}$ and $s \in F_{\beta_{j^{\prime}}}^{s_{j^{\prime}}\left(\tau^{\prime}\right)}$ for all $j^{\prime} \leq j, \tau^{\prime} \subseteq \tau$ and all $s \in T_{\tau}$. For each $j^{\prime} \leq j$, at most one $s \in T_{\tau}$ does not belong to $H_{\gamma_{j^{\prime}}}^{n_{j^{\prime}}}$. Since $2^{j+2} \geq j+3$, we can find $s_{j+1}(\tau \frown 0), s_{j+1}(\tau \frown 1) \in$ $T_{\tau} \cap \bigcap_{j^{\prime} \leq j} H_{\gamma_{j^{\prime}}}^{n_{j^{\prime}}}$, as required. Finally, let $n_{j+1}$ be such that $s_{j+1}(\tau) \in H_{\gamma_{j+1}}^{n_{j+1}}$ for all $\tau \in 2^{j+1}$. This completes the construction.

For $x \in 2^{\omega}$, define $y=y_{x}$ by $y\left\lceil i_{\ell_{j}}=s_{j}(x \nmid j)\right.$ for all $j$. Then $y \in$ $\bigcap_{j} F_{\beta_{j}}^{s_{j}(x \backslash j)} \cap \bigcap_{j} H_{\gamma_{j}}^{n_{j}} \subseteq \bigcap_{j} F_{\beta_{j}} \cap \bigcap_{j} H_{\gamma_{j}}$. Thus $\left\{y_{x}: x \in 2^{\omega}\right\} \subseteq \bigcap_{j} F_{\beta_{j}} \cap$ $\bigcap_{j} H_{\gamma_{j}} \subseteq X_{\alpha}$ is a perfect set. Since $X_{\alpha+1} \subseteq Y_{\alpha}, Y_{\alpha}$ is perfect as well.

In fact, a straightforward generalization shows that if $F \subseteq X_{\alpha}$ is a nonempty basic clopen set, then $F$ contains a perfect subset. Similarly for $Y_{\alpha}$.

For $x \in 2^{\omega}$ infinite (i.e. $x \in[\omega]^{\omega}$ ), let

$$
G_{x}=\{y: \text { there are infinitely many } l \text { such that } y(l)=x(l)=1\} .
$$

This is the set of all $y$ which have infinite intersection with $x$. Clearly, $G_{x}$ is a $G_{\delta}$ set. More explicitly, $G_{x}=\bigcap_{n \in \omega} G_{x}^{n}$, where

$$
G_{x}^{n}=\left\{y: \exists l_{0}, \ldots, l_{n-1} \text { distinct such that } y\left(l_{j}\right)=x\left(l_{j}\right)=1 \text { for } j<n\right\} .
$$

This is the set of all $y$ whose intersection with $x$ is of size at least $n$. Clearly, each $G_{x}^{n}$ is dense open in the standard topology of $2^{\omega}$. So $G_{x}$ is dense $G_{\delta}$.

Lemma 3.6. Assume $x$ does not belong to the ideal generated by $a_{\beta}$, $\beta<\alpha$. (That is, $x$ is not almost contained in a finite union of $a_{\beta}, \beta<\alpha$.) Then $G_{x}^{n}$ is dense open in the space $\left(Y_{\alpha}, \mathcal{T}_{\alpha}\right)$.

Proof. This is similar to the proof of Lemma 3.5. By 3.3, basic open sets of the topology $\mathcal{T}_{\alpha}$ are finite intersections of the form $\bigcap_{j<m_{0}} F_{\beta_{j}}^{s} \cap$ $\bigcap_{j<m_{1}} H_{\gamma_{j}}^{n_{j}} \neq \emptyset$, where $s \in 2^{<\omega}$ and $\beta_{j}, \gamma_{j}<\alpha$. By extending $s$ if necessary, we may assume $|s| \in I$ and, by Lemma 3.4 , if we let $i_{k}=|s|$ then $\mid\left(i_{k}, i_{k+1}\right) \backslash$ $\bigcup_{j<m_{0}} a_{\beta_{j}} \mid \geq(n+1) m_{1}$. Next choose $l_{0}, \ldots, l_{n-1} \in x \backslash \bigcup_{j<m_{0}} a_{\beta_{j}}$ and $l>$ $k+1$ with $i_{k+1} \leq l_{0}<l_{1}<\cdots<l_{n-1}<i_{l}$. Consider the set $T$ of all 
$t \supseteq s$ with $|t|=i_{l}, t\left(l_{j}\right)=1$ for all $j<n$ and $t(i)=0$ for all $i$ such that $i \neq l_{j}(j<n)$ and $i \notin\left(i_{k}, i_{k+1}\right) \backslash \bigcup_{j<m_{0}} a_{\beta_{j}}$.

Clearly, $|T| \geq 2^{(n+1) m_{1}}$. Also, $[t] \cap \bigcap_{j<m_{0}} F_{\beta_{j}}^{s} \neq \emptyset$ for all $t \in T$. By the definition of $H_{\gamma_{j}}^{n_{j}}$, it is easily seen that at most $n+1$ many $t \in T$ do not belong to the tree defining $H_{\gamma_{j}}^{n_{j}}$. Hence for at most $(n+1) m_{1}$ such $t \in T$, we may have $[t] \cap \bigcap_{j<m_{0}} F_{\beta_{j}}^{s} \cap \bigcap_{j<m_{1}} H_{\gamma_{j}}^{n_{j}}=\emptyset$. Since $2^{(n+1) m_{1}}>(n+1) m_{1}$, we can find $t \in T$ such that $[t] \cap \bigcap_{j<m_{0}} F_{\beta_{j}}^{s} \cap \bigcap_{j<m_{1}} H_{\gamma_{j}}^{n_{j}} \neq \emptyset$. Clearly, $[t] \subseteq G_{x}^{n}$. Thus, $\bigcap_{j<m_{0}} F_{\beta_{j}}^{t} \cap \bigcap_{j<m_{1}} H_{\gamma_{j}}^{n_{j}} \subseteq G_{x}^{n}$ and we are done.

Corollary 3.7. $\mathcal{A}=\left\{a_{\alpha}: \alpha<\omega_{1}\right\}$ is a MAD family.

Proof. Let $x \in 2^{\omega}$. We need to show that there is an $\alpha<\omega_{1}$ such that $\left|x \cap a_{\alpha}\right|=\aleph_{0}$. Without loss of generality, we may assume that $x$ does not belong to the ideal generated by the $a_{\alpha}$. (Otherwise, the proof is trivial.) Find $\alpha$ such that $x \in M_{\alpha}$. By the previous lemma, $G_{x}^{n}$ is dense open in $\left(Y_{\alpha}, \mathcal{T}_{\alpha}\right)$ for all $n \in \omega$. Since $a_{\alpha} \in Y_{\alpha}$ is Cohen-generic over $M_{\alpha}$, it follows immediately that $a_{\alpha} \in G_{x}^{n}$ for all $n \in \omega$. Thus, $a_{\alpha} \in \bigcap_{n \in \omega} G_{x}^{n}=G_{x}$. Hence, $\left|a_{\alpha} \cap x\right|=\aleph_{0}$.

3.3. The $G_{\delta}$ sets $U_{\alpha}$ witnessing that $\mathcal{A}$ is a $\sigma$-set (construction of $Y_{\alpha+1}$ ). We now consider the second part of the construction: the construction of the space $Y_{\alpha+1}$ and its associated objects.

Assume we have a list $\left\langle B_{\alpha}: \alpha<\omega_{1}\right\rangle$ of all Borel sets such that $B_{\alpha} \in N_{\alpha}$. In $N_{\alpha}, B_{\alpha} \cap X_{\alpha+1}$ has the property of Baire (because it is Borel) in the space $\left(X_{\alpha+1}, \mathcal{S}_{\alpha+1}\right)$. Therefore there are disjoint sets $P_{\alpha}$ and $O_{\alpha}$ with $P_{\alpha}$ closed and $O_{\alpha}$ open, such that $P_{\alpha} \cup O_{\alpha}=X_{\alpha+1}$ and $B_{\alpha} \cap P_{\alpha}$ is comeager, while $B_{\alpha} \cap O_{\alpha}$ is meager. Let $P_{\alpha}^{n}, O_{\alpha}^{n}$ be decreasing sequences of open sets in $\left(X_{\alpha+1}, \mathcal{S}_{\alpha+1}\right)$ such that $P_{\alpha}^{0}=\operatorname{int}\left(P_{\alpha}\right), O_{\alpha}^{0}=O_{\alpha}, P_{\alpha}^{n} \subseteq P_{\alpha}^{0}$ is dense, $O_{\alpha}^{n} \subseteq O_{\alpha}$ is dense, $\bigcap_{n \in \omega} P_{\alpha}^{n} \subseteq B_{\alpha}$, and $\bigcap_{n \in \omega} O_{\alpha}^{n} \cap B_{\alpha}=\emptyset$.

The forcing $\mathbb{P}$ consists of finite consistent sets $p$ of conditions of the form:

- $\left(n, a_{\beta}\right)$ where $\beta \leq \alpha$ and $a_{\beta} \notin B_{\alpha}$,

- $(n, s)$ where $s \in 2^{<\omega}$ and $|s| \in I$,

- $(n, F)$ where $F$ is a typical basic clopen subset of $\mathcal{S}_{\alpha+1}$ (see Observation 3.3),

such that:

- if $(n, s) \in p$ and $|s|=i_{l} \in I$ then there is $i \in\left(i_{l-1}, i_{l}\right)$ such that $s(i)=1$,

- for each $i \in I$ and $n \in \omega$ there is at most one $s$ with $(n, s) \in p$ and $|s|=i$,

- if $(n, s) \in p$ and $|s|=i_{l} \in I$ then $l \geq n$,

- if $\left(n, a_{\beta}\right) \in p$ then $\left(n, a_{\beta}\lceil m) \notin p\right.$ for all $m$, 
- if $(n, F) \in p$ then $F \cap X_{\alpha+1} \subseteq P_{\alpha}^{n}$,

- if $(n, F) \in p$ then there is $s$ such that $F \subseteq[s]$ and $(n, s) \in p$.

The ordering $\leq$ is by extension. That is, $q \leq p \Leftrightarrow q \supseteq p$. This is a modification of Silver's standard forcing notion for turning a given set into a relative $G_{\delta}$ (see [7, Section 5], see also [8]).

$\mathbb{P}$ is a countable forcing notion in $N_{\alpha}$. (Recall that $M_{\alpha}$ is countable in $N_{\alpha}$ and so is $M_{\alpha}\left[a_{\alpha}\right]$, which contains $X_{\alpha+1}$ etc.)

Let us first check that we can always extend conditions appropriately.

Lemma 3.8. Assume $a_{\beta} \notin B_{\alpha}$ and $p \in \mathbb{P}$. Then there are $n \in \omega$ and $q \leq p$ such that $\left(n, a_{\beta}\right) \in q$.

Proof. Choose $n$ sufficiently large that no $(n, s)$ appears in $p$ and let $q=p \cup\left\{\left(n, a_{\beta}\right)\right\}$.

Lemma 3.9. Assume $a_{\beta} \in B_{\alpha}, p \in \mathbb{P}$ and $n \in \omega$. Then there are $m \in \omega$ and $q \leq p$ such that $\left(n, a_{\beta}\lceil m) \in q\right.$.

Proof. First choose $m_{0}$ sufficiently large that:

- $a_{\beta}\left\lceil m_{0} \neq a_{\gamma}\left\lceil m_{0}\right.\right.$ for all $\gamma$ such that $\left(n, a_{\gamma}\right) \in p$,

- $m_{0} \geq|s|$ for all $s$ with $(n, s) \in p$,

- $m_{0} \geq i_{n}$.

Then find $i_{l-1}<i<i_{l}$ with $m_{0} \leq i_{l-1}$ such that $a_{\beta}(i)=1$. (This is possible because $I \in M_{0}$ and such $i \notin I$ must exist by Cohen-genericity.) Let $m=i_{l}$ and $q=p \cup\left\{\left(n, a_{\beta}\lceil m)\right\}\right.$. Clearly, all the requirements are satisfied.

Lemma 3.10. Assume $F \cap X_{\alpha+1} \subseteq P_{\alpha}^{n}$ is non-empty open (in the sense of $\left.\left(X_{\alpha+1}, \mathcal{S}_{\alpha+1}\right)\right)$ and $p \in \mathbb{P}$. Then there are $\emptyset \neq H \subseteq F$ and $q \leq p$ such that $(n, H) \in q$.

Proof. Shrinking $F$ if necessary, we may assume without loss that $a_{\beta} \notin F$ for all $\beta$ with $\left(n, a_{\beta}\right) \in p$. Again choose $m_{0}$ such that

- $\left[a_{\beta}\left\lceil m_{0}\right] \cap F=\emptyset\right.$ for all $\beta$ such that $\left(n, a_{\beta}\right) \in p$,

- $m_{0} \geq|s|$ for all $s$ with $(n, s) \in p$,

- $m_{0} \geq i_{n}$.

Then find $i_{l-1}<i<i_{l}$ with $m_{0} \leq i_{l-1}$ and $t \in 2^{<\omega}$ with $t(i)=1,|t|=i_{l}$ and $F \cap[t] \neq \emptyset$ (in $X_{\alpha+1}$ ). The argument showing there is such a $t$ is similar to, but easier than, the proof of Lemma 3.6. Let $H=F \cap[t]$ and let $q=p \cup\{(n, t),(n, H)\}$. It is easy to see that $q$ is indeed a condition and that $q \leq p$.

Let $G$ be $\mathbb{P}$-generic over $N_{\alpha}$ with $G \in M_{\alpha+1}$ (so $N_{\alpha}[G] \subseteq M_{\alpha+1}$ ). Such a $G$ clearly exists because $N_{\alpha}$ is countable in $M_{\alpha+1}$.

Set

$$
U_{\alpha}^{n}=\bigcup\{[s]: \exists p \in G((n, s) \in p)\}, \quad H_{\alpha}^{n}=2^{\omega} \backslash U_{\alpha}^{n} .
$$


Clearly, $U_{\alpha}^{n}$ is open in $2^{\omega}$ and $H_{\alpha}^{n}$ is closed in $2^{\omega}$. Also, $U_{\alpha}=\bigcap_{n \in \omega} U_{\alpha}^{n}$ is a $G_{\delta}$ set and $H_{\alpha}=\bigcup_{n \in \omega} H_{\alpha}^{n}$ is an $F_{\sigma}$ set (in the standard topology). It is immediate from the definition of the partial order $\mathbb{P}$ that the $U_{\alpha}^{n}$ and $H_{\alpha}^{n}$ satisfy all the stipulations required earlier.

Also set

$$
V_{\alpha}^{n}=\left(\bigcup\{F: \exists p \in G((n, F) \in p)\} \cap X_{\alpha+1}\right) \cup\left(O_{\alpha}^{n} \cap H_{\alpha}\right)
$$

and let $V_{\alpha}=\bigcap_{n} V_{\alpha}^{n}$.

Finally, as stipulated earlier,

$$
Y_{\alpha+1}=P_{\alpha} \cup\left(X_{\alpha+1} \cap H_{\alpha}\right)=P_{\alpha} \cup\left(O_{\alpha} \cap H_{\alpha}\right)
$$

and $\mathcal{T}_{\alpha+1}$ is the topology generated by $\mathcal{S}_{\alpha+1}$ and by sets of the form $F \cap H_{\alpha}^{n}$ where $F \cap X_{\alpha+1} \subseteq O_{\alpha}, F \in \mathcal{S}_{\alpha+1}$.

Corollary 3.11. $\forall \beta \leq \alpha\left(a_{\beta} \in U_{\alpha} \Leftrightarrow a_{\beta} \in B_{\alpha}\right)$.

Proof. $(\Rightarrow)$ This follows by Lemma 3.8 .

$(\Leftarrow)$ This follows by Lemma 3.9 .

Lemma 3.12. All $V_{\alpha}^{n}$ are dense open in $\left(Y_{\alpha+1}, \mathcal{T}_{\alpha+1}\right)$. Consequently, $V_{\alpha}$ is dense $G_{\delta}$ in $\left(Y_{\alpha+1}, \mathcal{T}_{\alpha+1}\right)$.

Proof. For $(n, F) \in p$ with $p \in G, F \cap X_{\alpha+1}$ is open in $\mathcal{S}_{\alpha+1}$ and thus in $\mathcal{T}_{\alpha+1}$. Also all $O_{\alpha}^{n} \cap H_{\alpha}^{m}, m \in \omega$, are open in $\mathcal{T}_{\alpha+1}$. Hence $V_{\alpha}^{n}$ is indeed open in $Y_{\alpha+1}$.

Therefore it suffices to show that the $V_{\alpha}^{n}$ are dense. Let $F \in \mathcal{T}_{\alpha+1}$ be non-empty. We need to show $V_{\alpha}^{n} \cap F \neq \emptyset$. Without loss of generality, we may assume $F \cap X_{\alpha+1} \subseteq P_{\alpha}$ or $F \cap X_{\alpha+1} \subseteq O_{\alpha}$. In the first case, we must have $F \in \mathcal{S}_{\alpha+1}$, by definition of $\mathcal{T}_{\alpha+1}$. By further shrinking $F$ if necessary, we may assume $F \cap X_{\alpha+1} \subseteq P_{\alpha}^{n}$. By Lemma 3.10 and genericity, there is a non-empty $H \subseteq F, H \in \mathcal{S}_{\alpha+1}$, such that $H \cap X_{\alpha+1} \subseteq V_{\alpha}^{n}$. Thus $V_{\alpha}^{n} \cap F \neq \emptyset$.

Therefore we may assume $F \cap X_{\alpha+1} \subseteq O_{\alpha}$. Then $F=F^{\prime} \cap \bigcap_{j<m} H_{\alpha}^{n_{j}}$ where $F^{\prime} \in \mathcal{S}_{\alpha+1}$ with $F^{\prime} \cap X_{\alpha+1} \subseteq O_{\alpha}$.

Work in the model $N_{\alpha}$, and assume $p \in \mathbb{P}$ forces $F^{\prime} \cap \bigcap_{j<m} \dot{H}_{\alpha}^{n_{j}} \neq \emptyset$. By 3.3, $F^{\prime}=\bigcap_{j<m_{0}} F_{\beta_{j}}^{s} \cap \bigcap_{j<m_{1}} H_{\gamma_{j}}^{k_{j}}$ with $\beta_{j}<\alpha+1$ and $\gamma_{j}<\alpha$. Without loss of generality $|s| \in I$. Since $F^{\prime} \subseteq[s]$, we must have $\left(n_{j}, s^{\prime}\right) \notin q$ for any $j<m, s^{\prime} \subseteq s$ and $q \leq p$. (This means that for each such $\left(n_{j}, s^{\prime}\right)$ with $\left|s^{\prime}\right|=i_{\ell} \in I$, either $\ell<n_{j}$ or $\left(n_{j}, t\right) \in p$ for some $t \neq s^{\prime}$ with $|t|=i_{\ell}$ or $s^{\prime} \uparrow\left(i_{\ell-1}, i_{\ell}\right)=0$ or $\left(n_{j}, a_{\beta}\right) \in p$ for some $\beta$ with $s^{\prime} \subseteq a_{\beta}$. Otherwise $q=p \cup\left\{\left(n_{j}, s^{\prime}\right)\right\} \leq p$, a contradiction.)

Let $s_{0} \supseteq s,\left|s_{0}\right| \in I$, be such that $s_{0}(i)=0$ for all $i$ with $|s| \leq i<\left|s_{0}\right|$ and $\left|s_{0}\right| \geq\left|s^{\prime}\right|$ for all $s^{\prime}$ with $\left(n_{j}, s^{\prime}\right) \in p$ for some $j$. By the definition of $\mathbb{P}$, $p$ still forces $\left[s_{0}\right] \cap F^{\prime} \cap \bigcap_{j<m} \dot{H}_{\alpha}^{n_{j}} \neq \emptyset$. (The point here is that no $\left(n_{j}, s^{\prime}\right)$ with $s^{\prime} \subseteq s_{0}$ and $|s|<\left|s^{\prime}\right| \leq\left|s_{0}\right|$ can belong to any $q \leq p$.) 
Since $O_{\alpha}^{n} \subseteq O_{\alpha}$ is open dense (in the topology $\mathcal{S}_{\alpha+1}$ ), we may find $\emptyset \neq$ $H^{\prime}=\bigcap_{j<m_{2}} F_{\beta_{j}}^{s_{1}} \cap \bigcap_{j<m_{3}} H_{\gamma_{j}}^{k_{j}} \subseteq\left[s_{0}\right] \cap F^{\prime}$ with $H^{\prime} \cap X_{\alpha+1} \subseteq O_{\alpha}^{n}$ where $m_{2} \geq m_{0}, m_{3} \geq m_{1}$, and $s_{0} \subseteq s_{1}$. Without loss of generality $\left|s_{1}\right| \in I$. Now strengthen $p$ to $q$ by adding appropriate conditions of the form $\left(n_{j}, s^{\prime}\right)$ with $s^{\prime} \nsubseteq s_{1},\left|s^{\prime}\right| \in I,\left|s_{0}\right|<\left|s^{\prime}\right| \leq\left|s_{1}\right|$ so as to guarantee that $\left(n_{j}, s^{\prime}\right) \notin r$ for any $j<m, s^{\prime} \subseteq s_{1}$ and $r \leq q$. This means that $q$ forces $H^{\prime} \cap \bigcap_{j<m} \dot{H}_{\alpha}^{n_{j}} \neq \emptyset$.

So, in the generic extension, we have $\emptyset \neq H^{\prime} \cap \bigcap_{j<m} H_{\alpha}^{n_{j}} \cap Y_{\alpha+1} \subseteq$ $F \cap O_{\alpha}^{n} \cap H_{\alpha}=F \cap V_{\alpha}^{n}$. This completes the proof of Lemma 3.12.

Corollary 3.13. $V_{\alpha} \cap P_{\alpha} \subseteq U_{\alpha} \cap B_{\alpha}$.

Proof. Clearly $V_{\alpha}^{n} \cap P_{\alpha} \subseteq P_{\alpha}^{n}$ by definition of the forcing. Since $\bigcap_{n \in \omega} P_{\alpha}^{n}$ $\subseteq B_{\alpha}$, it follows that $V_{\alpha} \cap P_{\alpha}=\bigcap_{n \in \omega}\left(V_{\alpha}^{n} \cap P_{\alpha}\right) \subseteq B_{\alpha}$. The definition of the forcing also gives $V_{\alpha}^{n} \cap P_{\alpha} \subseteq U_{\alpha}^{n}$. Thus, $V_{\alpha} \cap P_{\alpha} \subseteq U_{\alpha}$.

Corollary 3.14. $\left(V_{\alpha} \cap O_{\alpha}\right) \cap\left(B_{\alpha} \cup U_{\alpha}\right)=\emptyset$.

Proof. It is immediate from the definition that $V_{\alpha} \cap O_{\alpha}=\left(\bigcap_{n \in \omega} O_{\alpha}^{n}\right) \cap$ $H_{\alpha} \subseteq H_{\alpha}$. Since $U_{\alpha}=2^{\omega} \backslash H_{\alpha}$, it follows that $\left(V_{\alpha} \cap O_{\alpha}\right) \cap U_{\alpha}=\emptyset$. Also, $\bigcap_{n \in \omega} O_{\alpha}^{n} \cap B_{\alpha}=\emptyset$ so $\left(V_{\alpha} \cap O_{\alpha}\right) \cap B_{\alpha}=\emptyset$.

Corollary 3.15. $V_{\alpha}$ is dense $G_{\delta}$ in $\left(Y_{\alpha+1}, \mathcal{T}_{\alpha+1}\right)$ such that for all $x \in$ $V_{\alpha}, x \in U_{\alpha} \Leftrightarrow x \in B_{\alpha}$.

Proof. This is immediate from Lemma 3.12 and Corollaries 3.13 and 3.14 .

The point for having this result is that if we add $x$ to $Y_{\alpha+1}$ by Cohen forcing (e.g. if we add $a_{\alpha+1}$ ) then $x$ belongs to $U_{\alpha}$ if and only if it belongs to $B_{\alpha}$. So we can hope that Corollary 3.11 also holds for $\beta>\alpha$. However, for this we need that the denseness of $V_{\alpha}$ is preserved along the construction.

Lemma 3.16. For all $n \in \omega, \beta<\alpha, V_{\beta}^{n} \cap Y_{\alpha}$ is dense open in $\left(Y_{\alpha}, \mathcal{T}_{\alpha}\right)$ and $V_{\beta}^{n} \cap X_{\alpha+1}$ is dense open in $\left(X_{\alpha+1}, \mathcal{S}_{\alpha+1}\right)$.

Proof. Fix $\beta$ and $n$. The proof is by induction on $\alpha$.

Basic step: $\alpha=\beta+1$. Then $V_{\beta}^{n} \cap Y_{\alpha}=V_{\beta}^{n}$ and the claim for $Y_{\alpha}$ follows from Lemma 3.12.

For $X_{\alpha+1}$, argue as follows. Let $s \in 2^{<\omega}$ and let $F=\bigcap_{j<m_{0}} F_{\gamma_{j}}^{s} \cap$ $\bigcap_{j<m_{1}} H_{\delta_{j}}^{n_{j}} \neq \emptyset$ be a basic clopen set in $\left(Y_{\alpha}, \mathcal{T}_{\alpha}\right)$ where $\gamma_{j}, \delta_{j}<\alpha$ (see 3.3). Assume $|s| \in I$. We need to show that $F \cap F_{\alpha}^{s} \cap V_{\beta}^{n} \cap X_{\alpha+1} \neq \emptyset$.

Work in the model $M_{\alpha}$. Let $p=\bigcap_{j<k_{0}} F_{\epsilon_{j}}^{t} \cap \bigcap_{j<k_{1}} H_{\zeta_{j}}^{l_{j}} \neq \emptyset$ be a condition in the Cohen forcing in the space $\left(Y_{\alpha}, \mathcal{T}_{\alpha}\right)$. Assume $|t| \in I$. We need to find a stronger condition $q \leq p$ forcing that $F \cap \dot{F}_{\alpha}^{s} \cap V_{\beta}^{n} \cap \dot{X}_{\alpha+1} \neq \emptyset$.

If $|s| \geq|t|$ then let $s_{0}=s$. Otherwise, define $s_{0}$ as follows. Extend $s$ to $s_{0}$ with $\left|s_{0}\right|=|t|$ and $s_{0}(i)=0$ for all $i$ with $|s| \leq i<\left|s_{0}\right|$. Notice that by 
definition of the $F_{\gamma_{j}}^{s}$ and $H_{\delta_{j}}^{n_{j}}$, we must have $\left[s_{0}\right] \cap \bigcap_{j<m_{0}} F_{\gamma_{j}}^{s} \cap \bigcap_{j<m_{1}} H_{\delta_{j}}^{n_{j}}$ $\neq \emptyset$. That is, $\bigcap_{j<m_{0}} F_{\gamma_{j}}^{s_{0}} \cap \bigcap_{j<m_{1}} H_{\delta_{j}}^{n_{j}}$ is still basic open in $\left(Y_{\alpha}, \mathcal{T}_{\alpha}\right)$.

Since $V_{\beta}^{n} \cap Y_{\alpha}$ is dense open in $Y_{\alpha}$ (Lemma 3.12), we may find $\emptyset \neq H=$ $\bigcap_{j<m_{2}} F_{\gamma_{j}}^{s_{1}} \cap \bigcap_{j<m_{3}} H_{\delta_{j}}^{n_{j}} \subseteq\left[s_{0}\right] \cap F$ with $H \cap Y_{\alpha} \subseteq V_{\beta}^{n} \cap Y_{\alpha}$ where $m_{2} \geq m_{0}$, $m_{3} \geq m_{1}$ and $s_{0} \subseteq s_{1}$. Assume $\left|s_{1}\right| \in I$.

Extend $t$ to $t_{1}$ with $\left|t_{1}\right|=\left|s_{1}\right|$ such that $t_{1}(i)=0$ for all $i$ with $|t| \leq i<\left|t_{1}\right|$. Again by the definition of the $F_{\epsilon_{j}}^{t}$ and $H_{\zeta_{j}}^{l_{j}}, q=\left[t_{1}\right] \cap p=\bigcap_{j<k_{0}} F_{\epsilon_{j}}^{t_{1}} \cap$ $\bigcap_{j<k_{1}} H_{\zeta_{j}}^{l_{j}} \neq \emptyset$ is a condition strengthening $p$. Clearly, $q$ forces $H \cap \dot{F}_{\alpha}^{s_{1}} \neq \emptyset$. Since $t_{1}(i)=0$ for $|t| \leq i<\left|t_{1}\right|, q$ also forces $\dot{F}_{\alpha}^{s_{1}} \subseteq \dot{F}_{\alpha}^{s_{0}}$. Furthermore, since $s_{0}(i)=0$ for $|s| \leq i<\left|s_{0}\right|, q$ forces $\dot{F}_{\alpha}^{s_{0}} \subseteq \dot{F}_{\alpha}^{s}$.

So, in the generic extension, we have $\emptyset \neq H \cap F_{\alpha}^{s_{1}} \cap X_{\alpha+1} \subseteq F \cap F_{\alpha}^{s} \cap$ $V_{\beta}^{n} \cap X_{\alpha+1}$. This completes the basic step.

Induction step (successor): $\alpha=\alpha_{0}+1$. First deal with $Y_{\alpha}=Y_{\alpha_{0}+1}$. We assume $V_{\beta}^{n} \cap X_{\alpha}$ is dense open in $\left(X_{\alpha}, \mathcal{S}_{\alpha}\right)$. Let $F=F^{\prime} \cap \bigcap_{j<m} H_{\alpha_{0}}^{n_{j}}$ where $F^{\prime} \in \mathcal{S}_{\alpha}$. Work in the model $N_{\alpha_{0}}$ and repeat the second part of the argument of the proof of Lemma 3.12 with $O_{\alpha}^{n}$ replaced by $V_{\beta}^{n}$.

We leave the details to the reader.

The induction step for $X_{\alpha+1}=X_{\alpha_{0}+2}$ is like the basic step.

Induction step (limit): $\alpha$ is a limit ordinal. Then $X_{\alpha}=Y_{\alpha}=\bigcap_{\gamma<\alpha} X_{\gamma}=$ $\bigcap_{\gamma<\alpha} Y_{\gamma}$. If $F$ is a basic clopen in $\left(X_{\alpha}, \mathcal{S}_{\alpha}\right)$ then by construction there is $\gamma<\alpha$ such that $F$ is basic clopen in $\left(X_{\gamma}, \mathcal{S}_{\gamma}\right)$. Thus, there is a basic clopen $H \subseteq F$ with $\emptyset \neq H \cap X_{\gamma} \subseteq F \cap V_{\beta}^{n} \cap X_{\gamma}$ by the induction hypothesis. Now simply notice that $H \cap X_{\alpha} \neq \emptyset$ (see Lemma 3.5 and the comment after its proof), so we are done.

This completes the proof of Lemma 3.16 .

Corollary 3.17. $\forall \alpha, \beta<\omega_{1}\left(a_{\beta} \in U_{\alpha} \Leftrightarrow a_{\beta} \in B_{\alpha}\right)$.

Proof. For $\beta \leq \alpha$ this is simply Corollary 3.11. So assume $\beta>\alpha$. Fix $n$. By Lemma 3.16, $V_{\alpha}^{n} \cap Y_{\beta}$ is dense open in $Y_{\beta}$. Since $a_{\beta}$ is Cohen-generic in $Y_{\beta}$ over $M_{\beta}, a_{\beta} \in V_{\alpha}^{n}$ follows. Thus, $a_{\beta} \in \bigcap_{n \in \omega} V_{\alpha}^{n}=V_{\alpha}$. By Corollary 3.15, $a_{\beta} \in U_{\alpha} \Leftrightarrow a_{\beta} \in B_{\alpha}$.

Corollary 3.18. $\mathcal{A}=\left\{a_{\alpha}: \alpha<\omega_{1}\right\}$ is a $\sigma$-set.

Proof. By Corollary 3.17, $\mathcal{A} \cap B_{\alpha}=\mathcal{A} \cap U_{\alpha}$ for all $\alpha<\omega_{1}$. Since for every $\alpha<\omega_{1}, U_{\alpha}$ is a $G_{\delta}$ set, we conclude that every Borel set is a relative $G_{\delta}$ and we are done.

4. Generalizations. Theorem 2 can be generalized under the assumption that a large enough fragment of Martin's axiom MA holds. Say a set of 
reals $X \subseteq 2^{\omega}$ is c-concentrated on $Y \subseteq 2^{\omega}$ if for any open $U \supseteq Y$, we have $|X \backslash U|<\mathfrak{c}$ (see [6]).

TheOREM 4.1. Assume MA( $\sigma$-centered $)$. Then there is an infinite MAD family which is $\mathfrak{c}$-concentrated on a countable subset of itself.

Sketch of proof. This is like the proof of Theorem 2 in Section 2, but we need to replace the recursive construction of the $a_{\alpha}$ by a forcing argument.

As before, we assume $(\star)$ for $\left\langle a_{n}: n \in \omega\right\rangle$ and construct $a_{\alpha}, \alpha \geq \omega$, satisfying conditions (1) through (4). At stage $\alpha$, we consider the p.o. $\mathbb{R}$ which consists of pairs $\langle s, X\rangle$ where $s \in 2^{<\omega}$ and $X \subseteq\left\{a_{\beta}: \beta<\alpha\right\}$ is finite, ordered by $\langle t, Y\rangle \leq\langle s, X\rangle$ if $t \supseteq s, Y \supseteq X$, and $t(i)=0$ for all $|s| \leq i<|t|$ with $i \in \bigcup X$. This is the standard $\sigma$-centered forcing notion for adding a set almost disjoint from all $a_{\beta}, \beta<\alpha$. The arguments in the proof of Theorem 2 now translate to density arguments which show that, if the generic $a_{\alpha}$ meets all relevant dense sets, then it will satisfy conditions (1) through (4). Thus, using $\mathrm{MA}(\sigma$-centered $)$, the construction can be carried out.

We do not know whether Theorem 1 can be generalized as well.

Conjecture 4.2. Assume $\mathrm{MA}(\sigma$-centered $)$. Then there is a $M A D \sigma$ set.

The approach taken in Section 3 does not seem to generalize easily: if $\alpha \geq \omega_{1}$, the spaces $\left(X_{\alpha}, \mathcal{S}_{\alpha}\right)$ and $\left(Y_{\alpha}, \mathcal{T}_{\alpha}\right)$ would not be second-countable (and thus not Polish) anymore, and while this does not affect much Subsections 3.1 and 3.2 (Cohen forcing would have to be replaced by the $\sigma$ centered partial order $\mathbb{Q}$ consisting of conditions of the form $p=\bigcap_{j<m_{0}} F_{\beta_{j}}^{s} \cap$ $\bigcap_{j<m_{1}} H_{\gamma_{j}}^{n_{j}} \neq \emptyset$, of course), it does affect the argument at the beginning of Subsection 3.3: there we used the fact that $B_{\alpha} \cap X_{\alpha+1}$ has the property of Baire in the Polish space $\left(X_{\alpha+1}, \mathcal{S}_{\alpha+1}\right)$.

Also we do not know to what extent the assumption $\mathrm{MA}(\sigma$-centered $)$ can be weakened in Theorem 4.1.

\section{References}

[1] S. H. Hechler, Short complete nested sequences in $\beta N \backslash N$ and small maximal almostdisjoint families, General Topology Appl. 2 (1972), 139-149.

[2] A. S. Kechris, Classical Descriptive Set Theory, Springer, New York, 1995.

[3] P. B. Larson, The Stationary Tower. Notes on a Course by W. Hugh Woodin, Univ. Lecture Ser. 32, Amer. Math. Soc., Providence, 2004.

[4] R. Laver, On the consistency of Borel's conjecture, Acta Math. 137 (1976), 151-169.

[5] A. W. Miller, On the length of Borel hierarchies, Ann. Math. Logic 16 (1979), 233267. 
[6] A. W. Miller, Special subsets of the real line, in: Handbook of Set-Theoretic Topology, North-Holland, Amsterdam, 1984, 201-233.

[7] - Descriptive Set Theory and Forcing, Lecture Notes in Logic 4, Springer, Berlin, 1995.

[8] -, A MAD Q-set, Fund. Math. 178 (2003), 271-281.

The Graduate School of Science and Technology

Kobe University

Rokko-dai 1-1, Nada-ku

Kobe 657-8501, Japan

E-mail: brendle@kurt.scitec.kobe-u.ac.jp gregorypiper@yahoo.co.uk

Received 27 October 2005;

in revised form 16 August 2006 